\title{
La Teoría Crítica como género
}

\author{
Beatriz Caballero Rodríguez \\ Universidad de Canterbury, Nueva Zelanda
}

\section{Resumen}

Este artículo arguye que, dadas las particularidades y los objetivos de la Teoría Crítica de la Escuela de Frankfurt, las clasificaciones tradicionales resultan inapropiadas. A pesar de su carácter utópico, su género no es la literatura utópica, puesto que la Teoría Crítica se define por mucho más que su objetivo: la auto-realización del ser humano. De la misma manera, tampoco encaja cómodamente dentro de las etiquetas de ensayo o discurso académico. De hecho, encasillarla en cualquiera de estos géneros supondría una domesticación de su discurso que afectaría su impacto. La tesis que aquí defiendo es que la Teoría Crítica se debe considerar mucho más que un simple rótulo que agrupe el trabajo de los miembros de la Escuela de Frankfurt; se refiere a la naturaleza idiosincrática de su trabajo entendido como la combinación de su objetivo, metodología, contenido y estilo. Es precisamente el conjunto de estas características lo que debe ser considerado como un género en sí mismo. El discurso de la Teoría Crítica sólo puede situarse, entonces, en una posición de desafío y rebeldía frente a los patrones establecidos de pensamiento y expresión al reconocer su particularidad como género diferenciado.

Palabras clave: Teoría Crítica, Escuela de Frankfurt, género, Adorno, Horkheimer, Marcuse. 
Abstract

This article argues that, based on the distinctiveness and the aim of Critical Theory, traditional classifications are inappropriate. Existing genre classifications are insufficient and counterproductive. It is my contention that Critical Theory becomes more than a banner to group together the work of the members of the Frankfurt School; it refers to the idiosyncratic nature of their work in relation to the combination of their aim, their methodology, as well as its content and style. It is on account of the conjunction of these features that Critical Theory constitutes a genre in its own right. By acknowledging this, its discourse becomes empowered and empowering, for it situates itself in a position of challenge and defiance in reference to established patterns of thought and expression.

Keywords: Critical Theory, Frankfurt School, Genre, Adorno, Horkheimer, Marcuse.

G 1 término "Teoría Crítica”, acuñado por los miembros de la Escuela de Frankfurt durante sus estancias en el exilio cuando trabajaban en la Universidad de Columbia en Nueva York, empezó usándose en parte a modo de código. Como explica Douglas Kellner, se trataba de una práctica necesaria dado su contexto sociopolítico, "in order to cover over its commitment to Marxism in an environment that is quite hostile to a theory associated with socialist revolution and the Soviet Union" (1989: 44). A pesar de que inicialmente el término "teoría crítica" se usaba con referencia a la investigación de los miembros del Institut für Sozialforschung, y todavía está estrechamente asociado con él, su uso se ha ampliado para describir también una gama más amplia de trabajo de carácter crítico, muy variado en cuanto a intención y sustancia. Sin embargo, dado que a lo largo del presente artículo este término se referirá exclusivamente a la Teoría Crítica en sentido frankfurtia- 
no, considero más apropiado el uso de las mayúsculas para evitar ambigüedades. ${ }^{1}$

Con el propósito de demostrar que la Teoría Crítica constituye un género en sí mismo, este artículo comenzará por identificar cuáles son las características comunes que la Teoría Crítica comparte, para proseguir analizando su objetivo, contenido, metodología y estilo. Sólo pasaremos a explorar su relación con el género tras investigar la relevancia de las interconexiones que existen entre estos aspectos de la Teoría Crítica. Al llegar a este punto argumentaré que la integridad y coherencia de la Teoría Crítica sólo pueden conservarse al considerarla como un género por derecho propio, puesto que de no hacerlo significaría su asimilación dentro de patrones preexistentes de expresión y de racionalidad, lo cual en definitiva iría en detrimento del posible impacto de su discurso.

\section{¿Qué es la Teoría Crítica?}

Para reflexionar sobre la naturaleza de la Teoría Crítica es imprescindible comenzar por destacar su diversidad. Como explica Fred Rush:

[...] while it is characterized by certain shared core philosophical concerns, Critical Theory exhibits a diversity among its proponents that both contributes to its richness and poses substantial barriers to understanding its significance (2004: 6).

Así pues, las diferencias idiosincráticas y, a veces, sustanciales entre las obras de los miembros individuales del Instituto de Investigación Social deben entenderse como diferentes manifestaciones de

${ }^{1}$ Para más detalle respecto a los matices del uso de las mayúsculas, refiero al lector al artículo de Vicente Gómez, titulado "La Teoría Crítica en España. Aspectos de una recepción” (2009: 3-5). 
la Teoría Crítica. A pesar de esta diversidad, como indica Rush, hay ciertos conceptos centrales que todos los teóricos críticos comparten, entre los que se incluyen no sólo sus objetivos teóricos, sino también aspectos centrales de su contenido y estilo, puesto que una de las características de la Teoría Crítica es, precisamente, la interrelación de sus elementos, el vínculo intrínseco entre contenido y estilo, teoría y praxis. Con esto presente, vamos a explorar a continuación cuáles son los elementos centrales que intervienen.

La Teoría Crítica es ante todo una crítica de la sociedad capitalista avanzada. Su carácter distintivo reside, como argumenta Kellner, en que si bien la teoría tradicional reproduce acríticamente la sociedad existente; en contraste, la Teoría Crítica se esfuerza por transformarla (1989: 46). Para la Escuela de Frankfurt, el estudio de la ideología está vinculado con un proyecto de liberación. Por tanto, trata de proporcionar críticas y alternativas a la teoría social tradicional o convencional, así como una crítica de una amplia gama de ideologías (véase Kellner, 1989: 1, 44). Lo que es más, en lugar de centrarse en problemas sólo específicamente socioeconómicos y políticos, su crítica se dirige contra la racionalidad específica sobre la que se erige la sociedad occidental: la razón instrumental.

Aunque Horkheimer ya desarrolló este concepto en su publicación Eclipse of Reason (1947), la frase "razón instrumental" apareció por primera vez en Zur Kritik der instrumentellen Vernun$f t$ (1967), es decir, en la traducción al alemán del inglés original. Allí, Horkheimer argumenta que la razón — tal y como se ha ejercitado desde la Ilustración - se rige por criterios de explotación, productividad y rentabilidad, que se han convertido en fines en sí mismos (1973: 21). ${ }^{2}$ Por esta razón sostiene que, lejos de ser el tipo de razón interpersonal, crítica y reflexiva que alentaría el de-

\footnotetext{
${ }^{2}$ La primera edición de esta obra de Horkheimer salió a la luz en 1947, pero por razones de disponibilidad cito la edición que hizo Continuum en 1973.
} 
sarrollo del individuo y de la sociedad que habita, constituye una forma instrumental de racionalidad que en última instancia resulta ser irracional en la medida en la que lleva al individuo por rutas destructivas —en lugar de constructivas o creativas- Esta racionalidad, que equipara lo útil a lo racional, da como resultado la subyugación de la naturaleza y del individuo. Siguiendo el hilo de la crítica frankfurtiana, Stephen E. Bronner explica las consecuencias de este tipo de racionalidad en los siguientes términos:

Technology generated under capitalism and its peculiar form of instrumental or scientific thinking increasingly undermines the reflective exercise of subjectivity. Moral judgment is understood as becoming increasingly subordinate to instrumental thinking and, as a consequence, individuals appear threatened with the loss of their capacity for normative judgment (2002: 223).

Es precisamente debido a esta amenaza al juicio normativo del individuo y a sus consecuencias que se considera que la razón instrumental es esencialmente irracional. Dicho de otra manera, se trata de una evaluación basada en criterios morales. Más específicamente, la razón instrumental hace uso de la razón en el ámbito de los medios de comunicación, la economía y la vida cultural, con el propósito de ejercer control social. Ésta es la dinámica de pensamiento y de acción que la Teoría Crítica desvela y denuncia, abriendo así nuevas líneas de investigación y, en particular, metodologías diferentes de investigación filosófica, sociológica y socio-política.

Tal y como subraya Axel Honneth, el concepto "razón" se vuelve crucial para la crítica presentada por los miembros de la Escuela, quienes asumen que "the cause of the negative state of society is to be found in a deficit in social rationality" (2004: 339). Por consiguiente, Honneth arguye que a pesar de las diferencias entre el vocabulario utilizado por los distintos miembros del Ins- 
tituto —Horkheimer: "organización irracional"; Adorno: "mundo administrado"; Marcuse: "sociedad unidimensional", "tolerancia represiva"; y Habermas: "colonización del mundo-vida social"-, todos ellos se refieren a una racionalidad deforme en contraste con una supuesta racionalidad "intacta" que podría brindarle a cada individuo la oportunidad de desarrollo y realización personal (2004: 338-339). Como Alan How señala, "for them [Critical Theorists] Reason proper was an altogether bigger and more significant concept than mere reason. Part of their aim was to challenge what currently passed for reason and extend it into something more comprehensive" (2003: 6).

El concepto de "Razón", en sentido amplio, es, pues, clave para la Teoría Crítica, sin embargo, pese al papel fundamental de la Razón, la naturaleza específica y las características de esta racionalidad "intacta", o bien no está muy elaborada o bien cambia significativamente de un pensador a otro, compartiendo sólo la premisa central de que debería permitir la auto-actualización del individuo (véase Honneth, 2004: 341). Influenciados por Hegel, la única característica de esta auto-actualización que comparten los miembros del Instituto es que implica "the conviction that the self-actualization of the individual is only successful when it is interwoven in its aims - by means of generally accepted principles or ends - with the self-actualization of all the other members of society" (Honneth, 2004: 342). Se trata de una premisa que es compatible no sólo con su preocupación por la justicia social, sino, más esencialmente, con las premisas éticas que, aunque indefinidas, guían el pensamiento de los teóricos críticos.

Según Honneth, la estructura de su crítica se apoya en tres elementos clave que son centrales para su pensamiento. En primer lugar, la crítica de la razón instrumental, entendida como una racionalidad socialmente deficiente, es el resultado del núcleo ético implícito en la Teoría Crítica (2004: 338). Horkheimer, Marcuse, 
Adorno y Habermas comparten la convicción de que la actualización de la libertad individual requiere la adopción de una praxis común que sea más que el resultado de la mera coordinación de los intereses individuales. Por tanto, su objetivo es la "cooperative selfactualization [which] includes [...] the notion that subjects are not able to achieve a successful social life as long as they have not recognized the common core of value judgements that lies behind their respective individual interests" (2004: 343). Sin embargo, es importante destacar que, pese a esta motivación ética, los teóricos críticos rechazan la posibilidad de una teoría moral universal, pues sería injustificablemente limitadora. En segundo lugar, el capitalismo se identifica como la causa de la racionalidad deficiente que denuncian (2004: 338). Y en tercer lugar, su crítica establece una conexión intrínseca a la praxis, la cual resulta necesaria puesto que el objetivo es superar el sufrimiento social causado por esta racionalidad deficiente (2004: 338). Con tales premisas, la propia Teoría Crítica debe adoptar una racionalidad diferente para evitar ser presa de su propia crítica. Por lo tanto, la Teoría Crítica se considera a sí misma esencialmente diferente no sólo en su contenido teórico, sino, más radicalmente, también en su metodología.

Sin embargo, hay que enfatizar que aunque la Teoría Crítica subraya las insuficiencias y las peligrosas consecuencias de la razón instrumental, esto no significa que la Escuela de Frankfurt abogue por una renuncia a la razón. Aunque en relación con Marcuse, Peter-Uwe Hohendahl señala una importante distinción, entre diversos tipos de razón, que también está presente en el resto de los teóricos críticos: "in One-Dimensional Man (1964), Marcuse differentiated more clearly between reason as 'Vernunft' [reason as common sense] and 'instrumental reason', yet he continued to link reason and liberation - thereby also insisting on the revolutionary potential of reason" (2001: 7). Por tanto, la propia lógica de la crítica de la razón instrumental ya sugiere la necesidad de 
desarrollar una razón alternativa más amplia sobre la que cualquier investigación verdaderamente transformadora debería basarse. Por este motivo, en un esfuerzo por liberarse del marco de racionalidad de la razón instrumental, las características distintivas de la Teoría Crítica incluyen un enfoque en cuanto a contenido y metodología que, tomando prestada la terminología de Kellner, podríamos llamar "supradisciplinar" (1989: 7, 77). A esto hay que añadir la estrecha relación entre la teoría y la praxis, y entre el contenido y estilo, que confluye en el uso subversivo del lenguaje.

\section{Los objetivos de la Teoría Critica}

El primer punto de cohesión para los teóricos críticos es su agenda normativa. En palabras de Chambers: "Critical Theory has a normative agenda. Its stated interest is the emancipation of humanity from injustice" (2004: 221). Como apunta Honneth, para los críticos teóricos, el objetivo normativo de la sociedad debería posibilitar la auto-actualización de cada miembro mutuamente posible (véase 2004: 344). El hecho de que no es así es lo que en última instancia convierte los objetivos y prácticas de la sociedad en irracionales.

Así pues, a pesar de su diversidad, debe señalarse que la Teoría Crítica puede describirse como holística, en el sentido de que su metodología no sólo viene dictada por sus objetivos y su punto de vista ideológico, sino que todos ellos afectan también a su contenido de manera que se establece una fuerte interconexión entre los distintos elementos que la componen. Esta interconexión se puede observar en el concepto de "praxis" que está presente en la teoría a través de la importancia que adquiere la experiencia. Como Simone Chambers afirma, los objetivos de la Teoría Crítica son "to show the internal relationship between knowledge and experience [...], and to use the interconnectedness of knowledge and experience to 
break out of the given and project normative goals and ends" (2004: 221). Esta interconexión se hace evidente en cualquier intento por analizar cualquiera de estos aspectos por separado, puesto que tal separación es ajena a la epistemología de la Teoría Crítica y sólo puede tener lugar artificialmente; razón por la cual dicho análisis — si pretende preservar y transmitir esta interconexión - requerirá necesariamente una cierta cantidad de repetición.

\section{La Teoría Crítica como metodología, praxis y estilo}

La metodología y los objetivos de la Teoría Crítica fueron presentados, por primera vez, por Horkheimer en su ensayo "Traditionelle und Kritische Theorie" (1937) y por Marcuse en "Philosophie und Kritische Theorie" (1937). Aunque el ensayo de Horkheimer constituye una declaración de la estructura y objetivos de la Teoría Crítica, como Rush indica, "focusing on it alone provides a simplified and overly neat answer to the question of what is supposed to make Critical Theory critical" (2004: 11). Por otra parte, la metodología y los objetivos que Marcuse propone también están sujetos a cambio y evolución. Como el propio Marcuse destaca en Negations, "that most of this was written before Auschwitz deeply separates it from the present" (1968: xv). También en Negations, Marcuse explica:

At that time [1934-8], it was not yet clear that the powers that had defeated fascism by virtue of their technical and economic superiority would strengthen and streamline the social structure which had produced fascism. [...] Capitalist society had not yet revealed all its strength and all its rationality, and the fate of the labor movement was still 'uncertain' (1968: xi).

Por lo tanto, es evidente que la Teoría Crítica no sólo tiene diversas manifestaciones, sino que también es flexible, puesto que pretende 
abordar los problemas asociados con la ideología y la racionalidad dominantes en el momento en el que se ejerce la crítica.

Es posible observar, ya desde su incepción, que la metodología de los miembros de la Escuela de Frankfurt está íntimamente ligada a sus objetivos. En su ensayo "Philosophie und Kritische Theorie" (1937), Marcuse establece que la diferencia entre la filosofía y la Teoría Crítica es que mientras la primera se ocupa solamente de la razón pura, nunca puede concluir nada que no estuviera ya presente en esencia. Sin embargo, la Teoría Crítica está anclada en diferentes aspectos de las condiciones materiales para el pensamiento y la percepción, supuestamente proporcionando una plataforma genuina para las transformaciones sociales (Hohendahl, 2001: 5-6). En palabras de Marcuse, "truth that is more than the truth of what is can be attained and intended only in opposition to established social relationships" (1968: 149). Buscar esa verdad es uno de los objetivos principales de la Teoría Crítica.

\section{La metodología de la Teoría Crítica}

Como ya hemos dicho, la Teoría Crítica es un esfuerzo supradisciplinar por construir una teoría social integral que puede enfrentarse a los problemas sociales y políticos clave resultantes del capitalismo avanzado. Al adoptar un enfoque supradisciplinar, la Teoría Crítica procura integrar la experiencia y la realidad en su análisis teórico (véase Kellner, 1989: 7-8, 36). A pesar de este enfoque, la Teoría Crítica considera sospechosas las soluciones totalizadoras. Rechaza el pensamiento sistemático y reivindica el valor de las perspectivas fragmentarias, contradictorias e incluso experimentales de la realidad, ya que sólo al abarcar estos aspectos pueden asirse las complejidades de la realidad que se estudia.

En fuerte contraste con las tradiciones empírica, analítica y positivista del pensamiento anglófono, para la Teoría Crítica la 
especulación constituye un elemento esencial de la razón y, por consiguiente, de su metodología. How identifica y reivindica el valor positivo que tiene la especulación para la Teoría Crítica:

In everyday English usage, the word 'speculation' suggests something vague and probably unjustified [...]. But because Critical Theory always sought to bring the social sciences into conjunction with philosophy I discovered the role of speculation could be seen in a quite different light. [...] Marcuse [...] in particular explicitly drew out Hegel's ideas on the intrinsically speculative nature of reason to form the basis of Critical Theory's critique of empiricism (2003: 2-3).

Esto es así porque, como ya hemos visto, la Teoría Crítica se ocupa de las interpretaciones de lo que existe, de manera tal que la razón pueda llegar a conclusiones que vayan más allá de sus premisas iniciales. Rush lo explica en relación con Adorno:

Adorno [...] does not think that Critical Theory is a theory of interpretation in the ordinary sense. Critical Theory does not study its objects with the aim of revealing meanings that are already there, independent of the interpretive process [...]. The objects of interpretation, as well as any particular interpretation of them, are always subject to further interpretation (2004: 34).

Para lograrlo es necesario tener conciencia de las interconexiones entre los diferentes aspectos de la sociedad y del tejido de la propia realidad — de ahí su supradisciplinaridad_-, además de permitir la especulación como parte de su metodología. Horkheimer y Adorno exploran ese concepto de "interconexión" mediante su dialéctica negativa y el estilo aforístico de Adorno. Por otra parte, Marcuse, en especial, defiende el valor de la especulación porque, como How indica, "for Marcuse the positivist/empiricist [...] emphasis on the 
givenness of facts entails a distinctly conservative acceptance of things as they are" (2003:3). En contraste con esta aceptación, Marcuse argumenta que "the real field of knowledge is not the given facts about things as they are, but the critical evaluation of them as a prelude to passing beyond their given form" (1955: 145). Para conseguirlo es necesario incorporar o hacer hincapié en el papel de elementos tradicionalmente excluidos del ámbito de la razón: la experiencia personal, la subjetividad y, en última instancia, la praxis.

\section{La función de estilo}

Los diferentes elementos que forman la Teoría Crítica son parte del esfuerzo integral de los críticos teóricos por substraerse a sí mismos y a sus lectores lo suficiente de la ideología que domina la sociedad capitalista avanzada como para poder concebir y realizar su crítica, pero también por abrir la posibilidad de la liberación del individuo de dicha ideología. Por este motivo, el estilo que los críticos teóricos utilizan para comunicarse es una consecuencia deliberada de sus objetivos y de su propia ideología; es la cristalización de su metodología.

Este estilo, aunque idiosincrásico a cada uno de ellos, comparte los mismos objetivos, a saber, la expresión y comunicación de secuencias de ideas complejas e interrelacionadas, pero también la desestabilización de la razón instrumental que les rodea. Lo que es más, este estilo de expresión exige la participación activa del lector. El lector ya no es un sujeto pasivo que absorbe información, sino un agente activo que se esfuerza por desentrañar el significado del texto, de la ideología y de la sociedad de las que trata. Es por ello que muchos críticos como How expresan su sorpresa ante la descripción de la Teoría Crítica como popular e incluso populista. En relación con la lectura de El hombre unidimensional de Marcuse, How reflexiona lo siguiente: 
[...] the style of language was the plainest obstacle. Marcuse mostly did not write in short easily absorbable sentences, but in long, roving, muscular phrases where a sentence could last a whole paragraph and where the subject and object of the sentence seemed only distant cousins. [...] In dialectical fashion each clause reciprocally (re)defined the one that went before while simultaneously adding meaning to the one that came after. [...] It forced the reader to hold a variety of inter-related ideas together and allowed them to co-mingle and influence each other (2003: 2).

Este lenguaje desafiante no es de ninguna manera exclusivo de Marcuse, pues otros miembros de la Escuela también retan las limitaciones lingüísticas de la expresión lineal, y exigen el involucramiento del lector con el texto. Como Edward Said explica, Adorno también desarrolla su propio estilo idiosincrático, que dista mucho de ser de fácil lectura:

Adorno is exceptionally difficult to read, whether in his original German or in any number of translations. Jameson [in Late Marxism: Adorno, or, the Persistence of Dialectic (1990)] speaks very well about the sheer intelligence of his sentences, their incomparable refinement, their programmatically complex internal movement (2002: 201).

Debo enfatizar que la adopción de este estilo es una elección deliberada que obedece a razones metodológicas. Como resultado, tal y como indica Rush, muchas de las obras de Adorno "are selfconscious exercises in embodying the movement of ideas in negative dialectic in a style of philosophical writing" (2004: 35). Este estilo se hace todavía más palpable en su obra posterior que se caracteriza por su expresión poética y aforística, con la esperanza de que "might resist instrumental demands by stubborn insistence upon nonpurposive activity" (Honneth, 2004: 342). 
Al optar por tan intricadas formas de expresión, estos pensadores, por un lado, demandan atención e involucración heurística del lector en el texto, sin la cual hay poca esperanza de que pueda entenderse su contenido; por otro lado, aspiran a acceder y transmitir conceptos que, de otra manera, estarían fuera del alcance del discurso filosófico, sociológico o estético tradicional. Lo que es más, este nivel de participación del lector en el proceso de desentrañamiento de significado de textos de esta complejidad puede resultar en un desafío a sus estructuras de pensamiento preexistentes, así como también en el desarrollo de sus propias habilidades radicalmente críticas. De esta manera, se abre la posibilidad de cumplir la meta final de la Teoría Crítica: la liberación del individuo de la racionalidad opresora dominante.

Es precisamente debido a la naturaleza idiosincrática de este estilo, en función de sus peculiaridades estilísticas, pero también en cuanto a sus objetivos y a su conexión intrínseca con la metodología de la Teoría Crítica, por lo que cualquier forma de discurso que exhiba estas características debe considerarse como Teoría Crítica también con relación a su género.

\section{El papel de la praxis}

Desde la perspectiva de la Teoría Crítica hay un vínculo intrínseco entre la teoría y la praxis, que se hará evidente una vez que se ha superado la racionalidad distorsionada imperante (véase Honneth, 2004: 353). Dado que su objetivo es superar esta racionalidad distorsionada, el trabajo de los teóricos críticos requiere el resurgimiento y la incorporación de este vínculo.

En el caso específico de los miembros de la Escuela de Frankfurt, el elemento de la praxis puede observarse desde dos perspectivas diferentes. En primer lugar, un aspecto de su praxis puede encontrarse en sus elecciones biográficas - aunque en me- 
nor medida en el caso de Horkheimer- (véase Bronner, 2002: 220, 223-224). Esta correspondencia entre pensamiento y biografía es más visible en el caso de Marcuse, que no sólo fue una fuente de influencia, sino también de apoyo para la Nueva Izquierda. También Erich Fromm fue un constante defensor de la justicia social, luchó por los derechos humanos internacionales y la abolición de las armas nucleares. Adorno, por su parte, concedió decenas de entrevistas radiofónicas en un esfuerzo por llegar al público y transmitir sus puntos de vista. La importancia de estas actividades radica en que, en tanto que la praxis es praxis humana, ésta implica siempre un elemento de experiencia y subjetividad que — sin caer presa del relativismo - también están integrados en la Teoría Crítica. En segundo lugar, aunque la Escuela no se centró en vislumbrar o diseñar caminos concretos y positivos para la liberación del individuo, su trabajo todavía tiene importantes implicaciones prácticas, las cuales pueden observarse en el análisis que hace Kellner sobre la defensa de Marcuse del Gran Rechazo:

Marcuse [...] constantly advocated the 'Great Refusal' as the proper political response to any form of irrational repression, and indeed this seems to be at least the starting point for political activism in the contemporary era: refusal of all forms of oppression and domination, relentless criticism of all of all [sic] policies that impact negatively on working people and progressive social programs, and militant opposition to any and all acts of aggression against Third World countries. Indeed, in an era of 'positive thinking', [...] Marcuse's emphasis on negative thinking, refusal, and opposition provides at least a starting point and part of a renewal of radical politics in the contemporary era (1990: 236).

Otra importante característica de esta posición es que no es prescriptiva. Como explica Rush: "Marcuse does not think that Critical Theory can prescribe what precise changes should take place. 
Its role is limited to displaying the relevant possibilities" (2004: 29-30). Este carácter no prescriptivo también se aplica al resto de los miembros del Instituto, que proporcionan una crítica de los diferentes aspectos de la sociedad y de la racionalidad sin elaborar ni proponer un plan positivo de acción. A pesar de la falta de instrucciones específicas orientadas hacia la acción, el objetivo final de su crítica sigue siendo la liberación y la auto-actualización del individuo, lo cual constituye una aspiración claramente práctica. Además, la Teoría Crítica puede alentar y asesorar la acción política, teniendo así un efecto práctico, primero, por medio de sus esfuerzos de concienciación de las limitaciones de la organización socio-política y económica existentes y, segundo, al identificar e indicar otras posibilidades pertinentes. Sin embargo, para hacerlo de manera coherente es de suma importancia que su discurso no esté inmerso en el mismo marco de racionalidad que es objeto de sus críticas. En consecuencia, es preciso subrayar que su discurso va más allá de la estrecha interrelación que existe entre los objetivos, la metodología, la praxis y el estilo que caracterizan a la Teoría Crítica entendida como el contenido de las ideas y la crítica planteada por los miembros de la Escuela; la Teoría Crítica constituye su propio marco de racionalidad que alberga la existencia de esa conciencia y crítica. Del mismo modo, la manifestación textual de esa racionalidad no puede ser asimilada por los géneros existentes sin asfixiar su potencial subversivo. Por eso propongo que la Teoría Crítica debe ser reconocida como un género en sí misma.

\section{La relación entre la Teoría Crítica y el género}

El término "género", tomado del francés, significa "tipo, clase o clasificación", y de hecho se ha utilizado con el fin de clasificar la publicación de textos de diversa índole, tanto escritos como también visuales y auditivos. Por lo tanto, se refiere a las características 
comunes que nos permiten agrupar textos al considerarlos similares para efectos de clasificación. Como explica Linda Anderson: "the markers of genre can thus be used to insist on the resemblance to what is already known, and to organize and regulate the meanings of a text for the reader" (2001: 10). Por lo tanto, a fin de decidir si un texto pertenece o no a un género específico, en primer lugar es necesario averiguar si ese texto presenta los marcadores o patrones que se encuentran de manera consistente en un género determinado.

Los teóricos contemporáneos tienden a describir los géneros en términos de "familias de semejanzas" entre textos, en lugar de buscar rígidos paradigmas de clasificación para definir la naturaleza de un texto (véase Swales, 1990: 49). No obstante, el género es más que la mera reproducción o repetición de patrones establecidos de expresión. El resultado de este elemento de repetición crea ciertas expectativas en el lector. Paul Cobley apunta: "genre is not a set of textual features that can be enumerated; rather, it is an expectation" (2006: 41). La identificación de un texto determinado con un género específico implica una declaración implícita de similitudes entre los dos que da como resultado la creación de numerosas expectativas con respecto a la longitud del texto, el alcance de su contenido, el estilo en el que está escrito, la identidad de sus lectores e incluso su impacto, entre otras características. Por ello, la clasificación de un texto dentro de un género no es neutral. De hecho, desde una perspectiva marxista, el género puede considerarse como un instrumento de control social, ya que contribuye a la reproducción de la ideología dominante (véase Feuer, 1992: 145). En tanto que el género es una expectativa, el texto a clasificar queda vinculado con los patrones ya existentes de un género apropiadamente similar. Este fenómeno tiene un impacto en la recepción del texto, en especial en el proceso de elaboración de significado que acomete el lector, lo cual es particularmente 
problemático para la Teoría Crítica cuando se tiene en cuenta que uno de sus rasgos definitorios es precisamente su esfuerzo por ir más allá de la mera interpretación de la realidad y por convertirse en una fuerza transformadora. En cambio, su clasificación dentro de cualquier género tradicional puede limitar su impacto y, debido a ello, sus posibilidades.

Las aspiraciones y las intenciones del autor de un texto, en la medida en que se observan, deben considerarse también, junto con el texto en sí, como un producto. Esto es, en particular, relevante en el caso de la Teoría Crítica, porque, tal y como argumento más arriba, lo que proporciona cohesión a las diferentes manifestaciones de la Teoría Crítica es justamente el objetivo que comparten para su proyecto. Teniendo en cuenta los efectos homogeneizadores y reaccionarios de la clasificación de un texto dentro de un género determinado, la asignación de un texto elaborado por uno de los miembros de la Escuela de Frankfurt a cualquiera de los géneros que son producto de la racionalidad "deficiente" que critican parece no sólo incoherente, sino también incompatible con las intenciones subversivas y creativas de su proyecto. Dado que su trabajo constituye un ataque contra los patrones establecidos de racionalidad, así como un intento por superarlos, resultaría inapropiado, contraproducente e incluso engańoso clasificar sus escritos dentro de géneros ya establecidos, como podrían ser el ensayo o la biografía.

Sin embargo, no está claro que sea necesario, deseable o incluso posible desechar por completo el concepto de "género". Como dice Derrida, "un texte ne saurait appartenir à aucun genre" (1986: 264). A nivel semiótico, el género proporciona un marco de referencia y de comprensión útil, dentro del cual no sólo interpretamos, sino también producimos los textos (Fowler, 1989: 216). Por esta razón, en tanto que la obra de los teóricos críticos comparte los mismos objetivos y requiere un enfoque metodológico 
específico - la coherencia entre el contenido y estilo— su trabajo también constituye un género propio, es decir, el de Teoría Crítica. Tal como observa Jane Feuer: "a genre is ultimately an abstract conception rather than something that exists empirically in the world" (1992: 144). Es esta flexibilidad relativa de la naturaleza del género como concepción abstracta la que, en última instancia, le permite a la Teoría Crítica convertirse en un género por derecho propio, puesto que sus rasgos comunes son suficientemente distintivos y coherentes como para conferirle esa cohesión.

\section{Conclusión}

Dado que el criterio principal para la clasificación de un texto bajo un género u otro conlleva un cierto grado de características comunes, tanto en términos de contenido, estilo, formato o una combinación de los mismos, se constituiría la normalización de los textos de Teoría Crítica como parte del marco de racionalidad contra el que dirigen su crítica. Por tanto, el rechazo de las etiquetas de género tradicionales se convierte en un requisito previo para la posibilidad de que la Teoría Crítica pueda aspirar a tener vigencia más allá del ámbito de la teoría.

En contraste, el reconocimiento de la Teoría Crítica como un género por derecho propio establece las condiciones y expectativas necesarias para que mediante esta clasificación de sus textos se reincorpore y rehabilite su intención y contenido políticos. De esta manera, mediante esta re-contextualización se le devuelve tanto al texto como al lector la posibilidad de ejercer un pensamiento y una acción transformadores fuera del marco de racionalidad dominante. 


\section{Bibliografía}

Anderson, Linda, 2001, Autobiography, Routledge, Londres y Nueva York.

Bronner, Stephen Eric, 2002, Of Critical Theory and its Theorists, Routledge, Londres y Nueva York.

Chambers, Simone, 2004, "The Politics of Critical Theory", en The Cambridge Companion to Critical Theory (ed. de Fred Rush), Cambridge University Press, Cambridge, pp. 219-249.

Cobley, Paul, 2006, "Objectivity and Immanence in Genre Theory", en Genre Matters: Essays in Theory and Criticism (eds. de Garin Dowd, Lesley Stevenson y Jeremy Strong), Intellect, Bristol, pp. 41-54.

Derrida, Jacques, 1986, “La loi du genre”, en Parages, Galilée, París.

Feuer, Jane, 1992, "Genre Study and Television", en Channels of Discourse, Reassembled: Television and Contemporary Criticism (ed. de Robert Allen), Routledge, Londres, pp. 138-159.

Fowler, Alastair, 1989, "Genre", en International Encyclopedia of Communications (ed. de Erik Barnouw), Oxford University Press, Nueva York, vol. 2, pp. 215-217.

Gómez, Vicente, 2009, "La Teoría Crítica en España. Aspectos de una recepción", en Constelaciones. Revista de Teoría Crítica, núm. 1, s.e, s.l.e, diciembre, pp. 3-35.

Hohendahl, Peter-Uwe, 2001, "From the Eclipse of Reason to Communicative Rationality and Beyond", en Critical Theory: Current State and Future Prospects (ed. de Peter-Uwe Hohendahl y Jamie Fisher), Berghahn, Nueva York, pp. 3-28.

Honneth, Axel, 2004, "Social Pathology of Reason: On the Intellectual Legacy of Critical Theory", en The Cambridge Companion to Critical Theory (ed. de Fred Rush), Cambridge University Press, Cambridge, pp. 336-360. 
Horkheimer, Max, 1937, "Traditionelle und Kritische Theorie”, en Zeitschrift für Sozialforschung, núm. 6, s.e, s.l.e, pp. 245-294.

—_, 1947, Eclipse of Reason, Oxford University Press, Nueva York.

__, 1967, Zur Kritik der Instrumentellen Vernunft, Fischer Verlag, Frankfurt Main.

— 1973, Eclipse of Reason, Continuum, Nueva York.

How, Alan, 2003, Critical Theory, Palgrave Macmillan, Nueva York.

Kellner, Douglas, 1989, Critical Theory, Marxism and Modernity, Polity Press, Oxford.

— 1990, "From 1984 to One-Dimensional Man: Reflections on Orwell and Marcuse", en Current Perspectives in Social Theory, núm. 10, s.e, s.l.e, pp. 223-252.

Marcuse, Herbert, 1937, "Philosophie und Kritische Theorie", en Zeitschrift für Sozialforschung, núm. 6, pp. 631-647.

- 1955, Reason and Revolution. Hegel and the Rise of Social Theory, Routledge y Kegan Paul, Londres y U.S.A.

— 1968, Negations: Essays in Critical Theory (ed. de Douglas Kellner y trans. por Jeremy J. Shapiro), Penguin, Londres.

Rush, Fred, 2004, "Conceptual Foundations of Early Critical Theory", en The Cambridge Companion to Critical Theory (ed. de Fred Rush), Cambridge University Press, Cambridge, pp. 6-39.

Said, Edward, 2002, "Adorno as Lateness Itself", en Adorno: A Critical Reader (eds. de Nigel C. Gibson y Andrew Rubin), Blackwell, Oxford, pp. 193-208.

Swales, John, 1990, Genre Analysis, Cambridge University Press, Cambridge. 